\title{
Relationship between Expanded Disability Status Scale scores and the presence of temporomandibular disorders in patients with multiple sclerosis
}

\author{
Lucas Senra Corrêa Carvalho ${ }^{1}$, Osvaldo José Moreira Nascimento ${ }^{1}$, \\ Luciane Lacerda Franco Rocha Rodrigues ${ }^{2}$, Andre Palma Da Cunha Matta ${ }^{1}$
}

Correspondence: Dr. Lucas Senra Corrêa Carvalho Email: lucas.sccarvalho@bol.com.br

\author{
'Department Of Neurology, University Federal \\ Fluminense, Niterói, Brazil, \\ ${ }^{2}$ Department Of Graduate Program In \\ Temporomandibular Disorder, São Leopoldo Mandic, \\ Campinas,Brazil
}

\section{ABSTRACT}

Objectives: The objectives of this study were to assess the prevalence of temporomandibular disorders (TMDs) in patients with relapsing-remitting multiple sclerosis (MS) and to investigate whether an association exists between the presence of TMD symptoms and the degree of MS-related disability. Materials and Methods: In all, 120 individuals were evaluated: 60 patients with a diagnosis of relapsing-remitting MS and 60 age- and sex-matched controls without neurological impairments. A questionnaire recommended by the European Academy of Craniomandibular Disorders for the assessment of TMD symptoms was administered. For those who answered affirmatively to at least one of the questions, the RDC/TMD Axis I instrument was used for a possible classification of TMD subtypes. The Expanded Disability Status Scale (EDSS) was the measure of the degree of MS-related disability. Statistical Analysis Used: Fisher's exact test was used to analyze the data. ANOVA was used to detect significant differences between means and to assess whether the factors influenced any of the dependent variables by comparing means from the different groups. Results: The prevalence of TMD symptoms in patients with MS was $61.7 \%$ versus $18.3 \%$ in the control group (CG). A diagnosis of TMD was established for $36.7 \%$ in the MS group and 3.3\% in the CG $(P=0.0001)$. There were statistically significant differences between degrees of MS-related disability and the prevalence of TMD $(P=0.0288)$. Conclusions: The prevalence of both TMD and TMD symptoms was significantly greater in the MS group. EDSS scores and TMD prevalence rates were inversely related.

Key words: Multiple sclerosis, orofacial pain, temporomandibular disorder

\section{INTRODUCTION}

Temporomandibular disorders (TMDs) are a group of increasingly frequent disorders with multifactorial etiology. The most common risk factors are depression, pain elsewhere in the body, clenching, onychophagia, emotional and physical traumas, dental microtraumas,

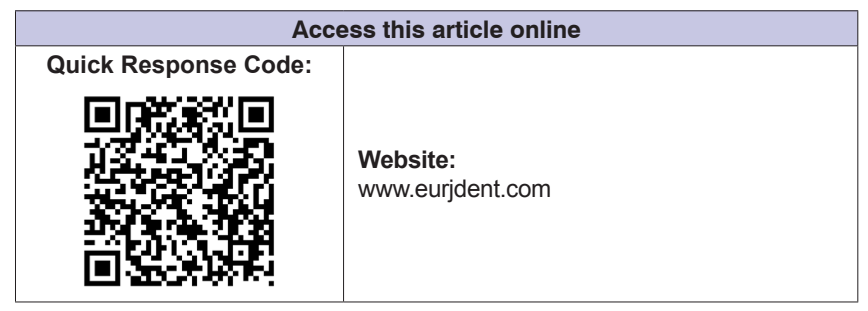

temporomandibular joint (TMJ) hypermobility, time-consuming dental treatments, somatic symptom disorders, and diseases compromising TMJ function. ${ }^{[1-4]}$ The condition comprises a group

This is an open access article distributed under the terms of the Creative Commons Attribution-NonCommercial-ShareAlike 3.0 License, which allows others to remix, tweak, and build upon the work non-commercially, as long as the author is credited and the new creations are licensed under the identical terms.

For reprints contact: reprints@medknow.com

How to cite this article: Carvalho LS, Nascimento OJ, Rodrigues LL, Matta AP. Relationship between Expanded Disability Status Scale scores and the presence of temporomandibular disorders in patients with multiple sclerosis. Eur J Dent 2018;12:144-8.

DOI: 10.4103/ejd.ejd_91_17 
of signs and symptoms reflecting alterations in TMJ function, mastication muscles, and related structures, and is mainly characterized by the presence of pain, joint sounds, and irregular or limited jaw function. ${ }^{[4-7]}$

Multiple sclerosis (MS) is a chronic, inflammatory, progressive, degenerative, and disabling neurological disease affecting the central nervous system. The disease is marked by attacks to the myelin sheath covering the axons, which are induced by specialized cells of the immune system -T-helper lymphocytes Types 1 and 17. For that reason, MS is considered an autoimmune disease, in which humoral immunity also plays a role as immunoglobulins are found in the cerebrospinal fluid of patients with MS. The pathogenesis of MS is heterogeneous, and its biological markers have not been elucidated to date. ${ }^{[8-11]}$ MS is predominantly found in populations of temperate and cold climate zones, with prevalence reaching 200/100,000 population. In Brazil, the prevalence of MS is around $15 / 100,000 \cdot{ }^{[12,13]}$ Some of the clinical manifestations of interest to dentistry are trigeminal neuralgia, facial palsy, trigeminal sensory neuropathy, and TMDs. ${ }^{[14,15]}$

Various studies ${ }^{[14-18]}$ have identified TMD in patients with MS. It has been hypothesized that cerebellar ataxia and proprioceptive alterations lead to increased susceptibility to TMD. However, there is a paucity of studies clarifying the relationship between TMD and MS or relating different degrees of MS disability based on the Expanded Disability Status Scale (EDSS) ${ }^{[19]}$ to TMD symptoms. The objectives of the present study were to assess the prevalence of TMD in patients with MS, compared those patients with a control group (CG), and determine the relationship between the degree of disability of patients with MS (EDSS) and the presence of TMD.

\section{MATERIALS AND METHODS}

The present investigation was approved by the Institutional Review Board of the University Federal Fluminense (UFF). It consisted of a cross-sectional, observational, controlled, and open study of 120 individuals at the Hospital University Antonio Pedro (HUAP/UFF), with and without MS, to determine the prevalence of TMD symptoms and possible subtypes in patients seen between January 2015 and August 2016. The MS group comprised 60 consecutive individuals with a diagnosis of relapsing-remitting type MS recruited from the HUAP-UFF neurology outpatient clinic. The study included male or female patients aged 18-80 years diagnosed according to the McDonald criteria ${ }^{[20]}$ as relapsing-remitting MS, with any EDSS score, who were receiving regular medical care at the neurology clinic and provided written informed consent. The CG, matched for sex and age, included 60 individuals attending the same outpatient clinic as chaperones or family members of the MS group participants. The control individuals had no diagnosis of MS or any neurological disease such as idiopathic inflammatory-demyelinating diseases and provided written informed consent.

Individuals of both groups were excluded if they declined to provide written informed consent or had cognitive deficits.

The investigation was conducted in one individual session in which the respondent's demographic data were recorded on a chart. The EDSS is the most used rating system to determine the severity and progression of MS. EDSS is based on functional systems as follows: pyramidal, cerebellar, brain stem, sensory, sphincters, visual, mental, and other. The EDSS score ranges from 0 to 10 , higher scores meaning more disability. ${ }^{[19]}$ The data pertaining to MS and respective neurological EDSS-based, impairment were verified by two or more of the clinic neurologists.

A questionnaire recommended by the $\mathrm{EACD}^{[21]}$ was used to identify TMD symptoms. The screening protocol includes four questions:

1. Do you have pain when you open your mouth wide or chew once a week or more?

2. Do you have pain in your temples, face, temporomandibular joint, or jaws once a week or more?

3. Have you recently registered that the jaw is locked or that you cannot open wide?

4. Do you have often headache more than once a week?

If the patient replies yes to one of the four questions, a more thorough history taking and assessment may be indicated.

The RDC/TMD Axis I ${ }^{[22]}$ instrument provided an important first step toward an etiology-based system. It was administered whenever an affirmative answer was given by individuals of either group (case or control) to at least one of the four screening questions. The questionnaires were administered by one of the investigators in charge of the study, exclusively. 
Fisher's exact test was used for the statistical analysis, with $2 \times 2$ contingency tables constructed to compare two nonparametric groups. ANOVA was used to detect significant differences between means and to assess whether the factors influenced any of the dependent variables by comparing means from the different groups. Statistical significance was set at $P<0.05$.

\section{RESULTS}

Gender distribution was 45 (75\%) females and 15 (25\%) males for both groups.

Age in the MS group ranged between 21 and 79 years (mean, $4.1 \pm 10.8$ years) versus $22-70$ years (mean, $43.7 \pm 11.93$ years) in the CG. There was no significant difference between groups (ANOVA, $P=0.75$ ).

TMD symptoms were significantly more frequent in the MS group (37 patients, 61.7\%) compared to the control (11 individuals, $18.3 \%$ ) (Fisher's exact, $P=0.0001$ ) [Tables 1-5].

A significant difference was found between groups (Fisher's exact, $P=0.0001$ ) with regard to the RDC/TMD assessment [Table 6]. Muscle-type TMD (myofascial pain with or without jaw opening limitation) accounted for $54.5 \%$ (12 patients), while joint-related TMD was found in $9.1 \%$ (2 patients), and the combined subtype in $36.4 \%$ (8 patients).

There was a statistically significant difference between the group with milder MS and the group with more severe disease (Fisher's exact, $P=0.0288$ ) with regard to the presence of TMD, as shown in Table 7 .

\section{DISCUSSION}

The groups in the present study were gender- and age-matched, with a mean age of approximately 43 years and $75 \%$ of females. Symptoms of TMD were noted in $61.7 \%$ of the MS group and only in $18.3 \%$ of the CG, while a diagnosis of TMD was made for $36.7 \%$ in the MS group and 3.3\% in the control. Myofascial pain TMD was diagnosed in $54.5 \%$ of patients in the MS group; the severity of MS was not proportional to the presentation of TMD in that group.

According to the literature, ${ }^{[14,17,18]}$ both MS and TMD are more prevalent in young adult females.

Symons et al. recorded the history and performed a clinical examination on 22 patients with MS;

\begin{tabular}{|c|c|c|c|}
\hline $\begin{array}{l}\text { Do you have pain when you open } \\
\text { your mouth wide or chew once a } \\
\text { week or more? }\end{array}$ & CG (\%) & MS (\%) & $P$ \\
\hline Yes & $3(5)$ & $7(11.7)$ & 0.3223 \\
\hline No & $57(95)$ & $53(88.3)$ & \\
\hline
\end{tabular}

\begin{tabular}{|c|c|c|c|}
\hline $\begin{array}{l}\text { Do you have pain your temples, } \\
\text { face, temporomandibular joint or } \\
\text { jaws, once a week or more? }\end{array}$ & CG (\%) & MS (\%) & $P$ \\
\hline Yes & $4(6.7)$ & $27(45)$ & $0.0001^{*}$ \\
\hline No & $56(93.3)$ & $33(55)$ & \\
\hline
\end{tabular}

$\begin{aligned} & \text { Table 3: Distribution of individuals according to the } \\
& \text { answer to the question } 3\end{aligned}$
$\begin{aligned} & \text { Have you lately registered that } \\
& \text { the jaw is locked or that you }\end{aligned}$
\begin{tabular}{llcc} 
can't open wide? & & & \\
\hline Yes & $2(3.3)$ & $9(15)$ & 0.0751 \\
No & $58(96.7)$ & $51(85)$ & \\
\hline
\end{tabular}

CG: Control group, MS: Multiple sclerosis

\begin{tabular}{|c|c|c|c|}
\hline $\begin{array}{l}\text { Do you have often headache } \\
\text { more than once a week? }\end{array}$ & CG (\%) & MS (\%) & $P$ \\
\hline Yes & $10(16.7)$ & $29(48.3)$ & 0.0002 \\
\hline No & $50(83.3)$ & $31(51.7)$ & \\
\hline
\end{tabular}

CG: Control group, MS: Multiple sclerosis

\begin{tabular}{|c|c|c|c|}
\hline Symptoms of TMD & CG (\%) & MS (\%) & $P$ \\
\hline Yes & $11(18.3)$ & $37(61.7)$ & $0.0001^{*}$ \\
\hline No & $49(81.7)$ & $23(38.3)$ & \\
\hline
\end{tabular}

however, the patient group was small, and there was no control. ${ }^{[17]}$ Kovac et al. evaluated a larger group: 50 patients with MS and 50 controls. ${ }^{[14]}$ Two studies reported only one case each, and neither was methodologically robust to confirm or dismiss a relationship between TMD and MS. ${ }^{[15,16]}$ Danesh-Sani et al. ${ }^{[18]}$ did not compare MS patients with a CG, yet they were able to study a considerably large sample: 500 patients with MS. The present study included a smaller sample (60 patients with MS and 60 controls) compared to that of Danesh-Sani et al. ${ }^{[18]}$ which can be 


\begin{tabular}{|c|c|c|c|}
\hline TMD & CG (\%) & MS (\%) & $P$ \\
\hline Yes & $2(3.3)$ & $\begin{array}{l}22(36.7) \\
\text { Myofascial pain } 12(54.5) \\
\text { Joint-related TMD } 2(9.1) \\
\text { Combined subtypes } 8(36.4)\end{array}$ & 0.0001 \\
\hline No & $58(96.7)$ & $38(63.3)$ & \\
\hline
\end{tabular}

\begin{tabular}{|c|c|c|c|c|}
\hline EDSS & $\begin{array}{l}\text { Diagnosis of } \\
\text { TMD (\%) }\end{array}$ & $\begin{array}{c}\text { Absence } \\
\text { of TMD (\%) }\end{array}$ & Total (\%) & $P$ \\
\hline $1.0-4.5$ & $\begin{array}{l}18(81.8) \\
\text { Myofascial pain } \\
9(40.9) \\
\text { Joint-related } \\
\text { TMD } 2 \text { ( } 9.1) \\
\text { Combined subtypes } \\
7(31.8)\end{array}$ & $20(52.6)$ & $38-63.3$ & $0.0288^{*}$ \\
\hline $5.0-10$ & $\begin{array}{l}4(18.2) \\
\text { Myofascial pain } \\
3(13.6) \\
\text { Combined subtypes } \\
1(4.6)\end{array}$ & $18(47.4)$ & $22-36.7$ & \\
\hline
\end{tabular}

explained by the difficulty in finding patients with MS in Brazil, given the very low prevalence rate compared to northern hemisphere countries.

Tweedle et al..$^{[16]}$ reported a case based on the patient's history and clinical examination including TMJ palpation. Badel et al. ${ }^{[15]}$ also reported a single case, in which they used RDC/TMD as the diagnostic tool. Symons et al. ${ }^{[17]}$ used history-taking followed by palpation of the TMJ and masticatory muscles for TMD diagnosis, but employed no validated criteria to establish the diagnosis. Kovac et al. ${ }^{[14]}$ and Danesh-Sani et al. ${ }^{[18]}$ conducted their assessments using the RDC/TMD, which is a validated instrument for TMD diagnosis. In the present study, in addition to the $\mathrm{RDC} / \mathrm{TMD}$, the EACD questionnaire, a standardized measure for TMD symptom assessment, and the EDSS, a measure of MS-related disability, were used. Neither was used in the aforementioned studies.

In the assessment of TMD symptoms, the present study showed a statistically significant difference between the MS and CGs, with the MS group manifesting more TMD symptoms compared to the control. It was found that $61.7 \%$ of the MS patients had at least one TMD symptom compared to only $18.3 \%$ in the CG.
These findings are comparable to those of the study by Symons et al., ${ }^{[17]}$ which identified TMD symptoms in $40.9 \%$ of the patients. However, their study had no CG. In the controlled study by Kovac et al., ${ }^{[14]}$ the difference was also significant, with $82 \%$ for the MS group and $24 \%$ for the control. Unlike the present study, those authors considered joint sounds as TMD symptoms, which could account for the difference between the two studies. This hypothesis was further supported by the fact that $30 \%$ of the patients with MS and $10 \%$ in the CG were found to have joint sounds in the study by Kovac et al. ${ }^{[14]}$ However, their study did not report the number of patients who manifested joint sounds in conjunction with other symptoms and how many had joint sounds like the only presenting symptom, which makes it difficult to determine the exact frequency of symptoms.

The study by Danesh-Sani et al. ${ }^{[18]}$ did not report which TMD symptoms were found.

In the present study, pain on jaw movements was noted in $11.7 \%$ of the patients with MS and $5 \%$ in the CG, whereas $31.8 \%$ of the patients in the study by Symons et al. ${ }^{[17]}$ had those symptoms. Kovac et al. ${ }^{[14]}$ reported pain during jaw movements in $22 \%$ of the MS group versus $4 \%$ in the CG.

Pain in the face, temples, TMJ, or maxilla occurred in $45 \%$ of the MS group and $6.7 \%$ in the CG. These findings are similar to those of Symons et al., ${ }^{[17]}$ as $40.9 \%$ of the patients with MS in their study complained of TMJ and facial pain, and to those of Kovac et al., ${ }^{[14]}$ who reported facial and TMJ pain in $54 \%$ of the patients with MS compared to $10 \%$ in the CG.

The present study identified closed lock in $15 \%$ of the MS group and 3.3\% of controls. These results are comparable to those of Kovac et al., ${ }^{[14]}$ who found closed lock in $22 \%$ of the patients with MS and $0 \%$ in the CG.

Headaches were found in $48.3 \%$ of the MS group and $16.7 \%$ controls in the present study. No other study reported that symptom.

It is worth noting that the presence of TMD symptoms alone does not necessarily translate into a diagnosis of the disorder. In the present study, TMD was diagnosed in $36.7 \%$ of the patients with MS and 3.3\% of controls-comparable rates to those found by Kovac et al., ${ }^{[14]}$ as $44.4 \%$ in their study were given a diagnosis of TMD in the MS group and $4 \%$ in the CG. In the study by Danesh-Sani et al., ${ }^{[18]} 58.2 \%$ of the patients 
had TMD. Regarding the TMD subtype in patients with MS, Kovac et al..$^{[14]}$ reported a $72 \%$ prevalence of TMD with myofascial pain. In the present study, $54.5 \%$ had myofascial pain involvement exclusively, while joint-related TMD was found in $9.1 \%$, and combined TMD accounted for $36.4 \%$ of cases. The other studies made no mention of TMD subtypes.

None of the referenced studies related the degree of disability from MS based on the EDSS to the presence of TMD. In the present study, there was a significant difference in EDSS scores for patients with TMD $(P=0.0288)$. It was found that the lower the EDSS, the greater the prevalence of TMD.

One limitation of the present study was the sample size, as a larger sample could allow for more robust results. Another shortcoming was the lack of follow-up, as is true for all cross-sectional studies.

A more thorough analysis evaluating the scores for each function on the EDSS and relating those scores to the presence of TMD could enable further elucidation.

Finally, the inclusion of other CGs such as patients with the primary progressive or secondary progressive type of MS would also open the way to major insights on this subject.

\section{Financial support and sponsorship}

Nil.

\section{Conflicts of interest}

There are no conflicts of interest.

\section{REFERENCES}

1. Huang GJ, Rue TC. Third-molar extraction as a risk factor for temporomandibular disorder. J Am Dent Assoc 2006;137:1547-54.

2. Ohrbach R, Dworkin SF. The evolution of TMD diagnosis: Past, present, future. J Dent Res 2016;95:1093-101.

3. List $\mathrm{T}$, Jensen RH. Temporomandibular disorders: Old ideas and new concepts. Cephalalgia 2017;37:692-704.

4. Restrepo CC, Medina I, Patiño I. Effect of occlusal splints on the temporomandibular disorders, dental wear and anxiety of Bruxist children. Eur J Dent 2011;5:441-50.
5. Lila-Krasniqi ZD, Shala KSh, Pustina-Krasniqi T, Bicaj T, Dula LJ, Guguvčevski L, et al. Differences between centric relation and maximum intercuspation as possible cause for development of temporomandibular disorder analyzed with T-scan III. Eur J Dent 2015;9:573-9.

6. LeResche L. Epidemiology of temporomandibular disorders: Implications for the investigation of etiologic factors. Crit Rev Oral Biol Med 1997;8:291-305.

7. Sonnesen L, Bakke M, Solow B. Temporomandibular disorders in relation to craniofacial dimensions, head posture and bite force in children selected for orthodontic treatment. Eur J Orthod 2001;23:179-92.

8. Compstom A, Lassmann H, Mcdonald I. The story of multiple sclerosis. Section 1. In: McAlpine`s Multiple Sclerosis. $4^{\text {th }}$ ed. ISBN 13: 9780443072710: Churchill Livingstone Elsevier Inc.; 2006. p. 3-68.

9. Handel AE, Giovannoni G, Ebers GC, Ramagopalan SV. Environmental factors and their timing in adult-onset multiple sclerosis. Nat Rev Neurol 2010;6:156-66.

10. Stangel M, Fredrikson S, Meinl E, Petzold A, Stüve O, Tumani H, et al. The utility of cerebrospinal fluid analysis in patients with multiple sclerosis. Nat Rev Neurol 2013;9:267-76.

11. Ciccarelli O, Toosy AT. Conversion from clinically isolated syndrome to multiple sclerosis: A large multicentre study. Mult Scler 2015;21:967-8.

12. Julien J, Ferrer X. Multiple sclerosis: An overview. Biomed Pharmacother 1989;43:335-46.

13. Fragoso YD, Peres M. Prevalence of multiple sclerosis in the city of Santos, São Paulo. Rev Bras Epidemiol 2007;10:479-82.

14. Kovac Z, Uhac I, Buković D, Cabov T, Kovacević D, Grzić R, et al. Oral health status and temporomandibular disorders in multiple sclerosis patients. Coll Antropol 2005;29:441-4.

15. Badel T, Carek A, Podoreski D, Pavicin IS, Lovko SK. Temporomandibular joint disorder in a patient with multiple sclerosis--review of literature with a clinical report. Coll Antropol 2010;34:1155-9.

16. Tweedle JA, Morrissey JB, Rankow RM. Mistaken TMJ pathology in unrecognized multiple sclerosis: Report of case. J Oral Surg 1970;28:785-8.

17. Symons AL, Bortolanza M, Godden S, Seymour G. A preliminary study into the dental health status of multiple sclerosis patients. Spec Care Dentist 1993;13:96-101.

18. Danesh-Sani SA, Rahimdoost A, Soltani M, Ghiyasi M, Haghdoost N, Sabzali-Zanjankhah S, et al. Clinical assessment of orofacial manifestations in 500 patients with multiple sclerosis. J Oral Maxillofac Surg 2013;71:290-4.

19. Kurtzke JF. Rating neurologic impairment in multiple sclerosis: An expanded disability status scale (EDSS). Neurology 1983;33:1444-52.

20. Polman CH, Reingold SC, Banwell B, Clanet M, Cohen JA, Filippi M, et al. Diagnostic criteria for multiple sclerosis: 2010 revisions to the mcDonald criteria. Ann Neurol 2011;69:292-302.

21. De Boever JA, Nilner M, Orthlieb JD, Steenks MH, Educational Committee of the European Academy of Craniomandibular Disorders. Recommendations by the EACD for examination, diagnosis, and management of patients with temporomandibular disorders and orofacial pain by the general dental practitioner. J Orofac Pain 2008;22:268-78.

22. Dworkin SF, LeResche L. Research diagnostic criteria for temporomandibular disorders: Review, criteria, examinations and specifications, critique. J Craniomandib Disord 1992;6:301-55. 